\title{
OP-Planung, OP-Management
}

\author{
René Waurick
}

\section{Zusammenfassung}

Die OP-Planung und -Dokumentation in elektronischen Krankenhausinformationssystemen trägt dazu bei, die patienten-, diagnostik- und therapieassoziierten Informationen den am perioperativen Prozess beteiligten Mitarbeitern unmittelbar verfügbar $\mathrm{zu}$ machen. Die Daten der OP-Planung und -Dokumentation sowie deren $\mathrm{Ab}$ weichungsanalyse bilden die Grundlage eines kennzahlenorientierten Berichtswesens bis hin zur tagesaktuellen Online-Version. Die strukturierte, interdisziplinäre und berufsgruppenübergreifende Kommunikation dieser Leistungs- und Kennzahlen ist die Voraussetzung für im Konsens zu vereinbarende Ziele, die an diesen Kennzahlen überprüft werden können. Teilhabe an Information und Kommunikation sind Grundlage einer vertrauensvollen Zusammenarbeit im OP, die einen kontinuierlichen Verbesserungsprozess und damit eine wirtschaftlich erfolgreiche Zusammenarbeit ermöglicht.

\section{Operating Room Scheduling, Operating Room Management}

Operating room scheduling and documentation in electronic hospital information systems offer open access for medical staff to patient's data as far as clinical diagnostics and therapy are concerned. Data of operating room scheduling and the corresponding deviation analysis allow a key-figurebased reporting system through to a continuously updated online version. The structured, interdisciplinary and comprehensive communication of performance numbers is the prerequisite for an agreement on objectives. Further monitoring of the key numbers is necessary to survey these objectives. All professional categories should take part in information and communication. This is a basic principle for a reliable cooperation in the operating rooms which enables a continuous improvement process and leads to economic success.

\section{Einleitung}

Der OP-Bereich ist regelhaft der kostenintensivste Bereich eines Krankenhauses. Die räumliche und technische Infrastruktur, Geräte und Instrumente, sowie insbesondere der hohe Personalaufwand verursachen diese hohen Kosten. Aus Sicht jeder Krankenhausleitung ist es daher von großer Bedeutung, dass die zur Verfügung stehende OP-Zeit möglichst effizient genutzt wird. Der Begriff „Effizienz" in diesem betriebswirtschaftlichen Zusammenhang bedeutet, mit dem gegebenen Aufwand einen möglichst

OP-JOURNAL 2014; 30: 28-32

(C) Georg Thieme Verlag KG Stuttgart · New York DOI http://dx.doi.org/10.1055/s-0034-1368270

großen Ertrag zu generieren. Aber nicht nur diese betriebswirtschaftliche Betrachtungsweise fordert gute Prozesse und funktionierende Abläufe. Für die $\mathrm{Zu-}$ friedenheit der im und um den OP herum tätigen Mitarbeiter aller Berufsgruppen - OP- und Anästhesie-Pflegedienst sowie Chirurgen, Anästhesisten und die unterstützenden Mitarbeiter aus der Instrumentenaufbereitung und Logistik - sind eine gute OP-Planung, eine effiziente Ablauforganisation und auch das Wissen, wirtschaftlich erfolgreich zu arbeiten, von großer Bedeutung. Die von uns betreuten Patienten merken natürlich ebenfalls, ob die Abläufe funktionieren oder ob Wartezeiten und Unstimmigkeiten zwischen den Mitarbeitern auftreten. Diese Eindrücke werden nach außen kommuniziert und prägen das Bild eines Krankenhauses in der Öffentlichkeit.

Gute Prozesse und funktionierende Abläufe beeinflussen auch die Zufriedenheit von Patienten und Mitarbeitern.

\section{OP-Planung}

Seit der Einführung der elektronischen Datenverarbeitung in allen Bereichen des Krankenhauses und der flächendeckenden Einführung von Krankenhausinformationssystemen, die von jedem Computerarbeitsplatz Zugriff auf alle mit einem Patienten und seiner Behandlung assoziierten Daten und Terminen erlauben, ist der OP-Plan, der als Zettel an der Wand hängt, nicht mehr zeitgemäß. Als gegenwärtige „best practice" muss angesehen werden, dass bereits bei der Indikationsstellung und Terminvereinbarung mit dem Patienten durch den behandelnden Arzt und/oder ein Case-Management die Operation in einen elektronischen OP-Plan eingetragen wird. Ob es sich zu diesem Zeitpunkt um abstrakte OP-Zeitkontingente oder bereits um konkrete OP-Säle handelt, spielt eine untergeordnete Rolle.

Die OP-Dauer für definierte Eingriffe kann und sollte im System hinterlegt sein, muss aber ggf. patientenspezifisch angepasst werden (können).

Während die Möglichkeit, für definierte Operationen die benötigten Instrumente, Geräte und Verbrauchsmaterialien in einem elektronischen OP-Plan zu hinterlegen, ebenfalls als Standard angesehen werden muss, ist die automatisierte Abfrage der Verfügbarkeit in der Instrumentenaufbereitung oder Lagerwirtschaft und Logistik für die meisten Krankenhäuser heute noch nicht möglich. Dazu ist es erforderlich, den Aufenthaltsort $z$. B. eines jeden OP-Siebes z. B. durch entsprechendes Scannen oder mit RFID (radio-frequency identification) kontinuierlich nachzuhalten und mit den Infor- 


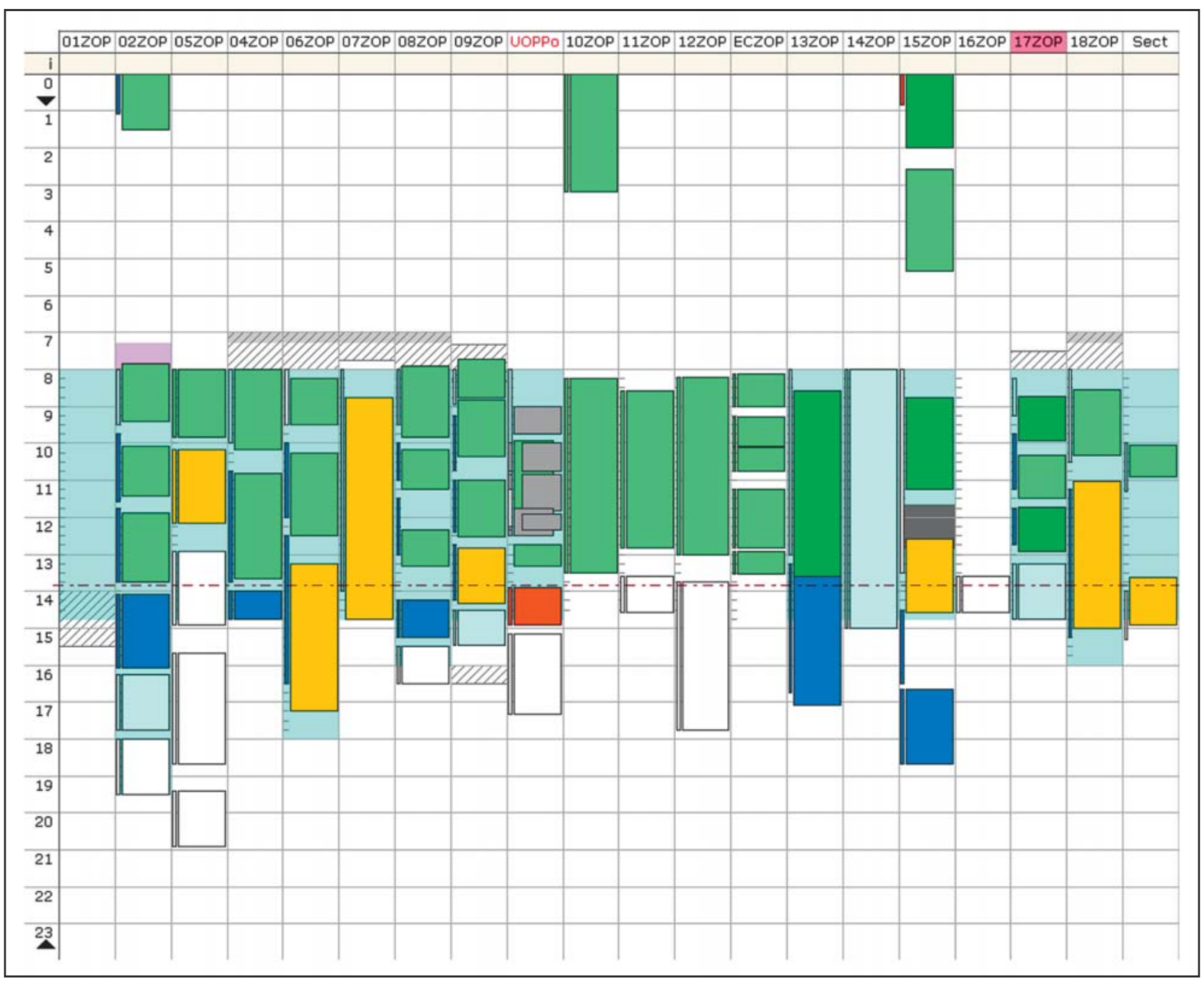

Abb. 1 Anonymisierte Übersicht über die 17 Säle des Zentral-OPs, die urologische Poliklinik, die „Elektrochirurgie“ (Herzschrittmacherimplantation u.ä.) und den Sectio-OP des UKM an einem Werktag um 13.50 Uhr.

mationen der Instrumentenaufbereitung und des OP-Plans zu korrelieren. In der Praxis würde z.B. der im OP-Plan eingetragene Operateur eine automatische E-Mail erhalten, wenn heute ein Sieb benutzt wird, das übermorgen um 8:00 Uhr für seine Operation benötigt wird, von der Instrumentenaufbereitung aber erst um 12:00 Uhr wieder zur Verfügung gestellt werden kann. Selbstverständlich können die Informationswege individuell gestaltet werden und die hier exemplarisch aufgeführte Siebverfügbarkeit auch an den OP-Pflegedienst gesendet oder direkt im OP-Plan angezeigt werden. Entsprechende Plausibilitätskontrollen können gleichermaßen für die Personaldisposition etabliert werden, sodass z. B. ein automatisierter Abgleich der Einteilung der Operateure mit Urlaubs- und Abwesenheitsplänen erfolgt.
Die an einer Operation beteiligten Mitarbeiter müssen ihrer individuellen Aufgabe entsprechend permanenten Zugriff auf Informationen zu dem einzelnen Patienten, seiner Erkrankung und seinem Behandlungsverlauf haben.

Idealerweise kann direkt aus dem OPPlan die Patientenakte geöffnet und auf sämtliche Informationen zugegriffen werden.

So stehen z.B. Röntgenbilder und andere bildgebende Diagnostik, deren Befunde und die der Labormedizin und Mikrobiologie, eingescannte OP- und Narkoseeinwilligungen sowie Arztbriefe online zur Verfügung.

Lese- und Schreibrechte in elektronischen Krankenakten können individuell zugewiesen werden, wobei aus Grün- den des Datenschutzes darauf zu achten ist, dass die Einsicht in personenbezogenen Daten von Patienten nur den Mitarbeitern möglich sein darf, die in einem unmittelbaren Behandlungszusammenhang stehen. So darf von Rechts wegen eine OP-Pflegekraft, die z.B. ausschließlich in der Gynäkologie eingesetzt ist, nicht Patientendaten des herzchirurgischen OP-Plans einsehen können. Die Einhaltung solcher datenschutzrechtlichen Regelungen kann in der Praxis schwierig sein und darf natürlich auch nicht zum Erliegen der Arbeitsfähigkeit eines OP-Bereichs führen.

Die OP-Dokumentation in einem Krankenhausinformationssystem erlaubt über die Eingabe von Zeitmarken, den Verlauf der einzelnen Operation, aber auch den eines Arbeitstags in einem OPSaal oder einem OP-Trakt in Echtzeit 
Tab. 1 Mögliche Aufgaben des OP-Managements.

\begin{tabular}{|c|c|c|c|c|}
\hline strategisches Management & Zukunftskonzepte & & & \\
\hline infrastrukturelles Management & Schnittstellen & Personalentwicklung & Wartung, Bau, Reparatur & \\
\hline Finanzmanagement & Budget-Controlling & & & \\
\hline $\begin{array}{l}\text { Qualitäts-u. Leistungs- } \\
\text { management }\end{array}$ & $\begin{array}{l}\text { Monitoring von Plan- } \\
\text { u. leistungszielen }\end{array}$ & $\begin{array}{l}\text { Kennzahlen, Darstellung } \\
\text { der Leistungsentwicklung, } \\
\text { Kontrollsystem }\end{array}$ & QM-Standards & $\begin{array}{l}\text { Belohnungs- } \\
\text { und Anreiz- } \\
\text { system }\end{array}$ \\
\hline Kapazitätsmanagement & $\begin{array}{l}\text { OP-Planung mit Notfall- } \\
\text { u. Ausfallmanagement }\end{array}$ & $\begin{array}{l}\text { Material- und Geräte- } \\
\text { disposition }\end{array}$ & $\begin{array}{l}\text { Kontrolle und Steuerung } \\
\text { der Planungsdaten }\end{array}$ & \\
\hline Patientenmanagement & $\begin{array}{l}\text { Steuerung der patienten- } \\
\text { bezogenen Abläufe }\end{array}$ & & & \\
\hline Personalmanagement & $\begin{array}{l}\text { Informationshoheit über } \\
\text { personelle Ressourcen }\end{array}$ & Feedbackgespräche & & \\
\hline $\begin{array}{l}\text { Kommunikations, Informa- } \\
\text { tions- u. Konfliktmanagement }\end{array}$ & $\begin{array}{l}\text { Entscheidungsfindung im } \\
\text { Konfliktfall }\end{array}$ & $\begin{array}{l}\text { Datenauswertung, Statistik, } \\
\text { Berichte und Informationen }\end{array}$ & OP-Besprechungen & \\
\hline sonstiges & $\begin{array}{l}\text { Planung, Entwicklung, } \\
\text { Projektarbeit }\end{array}$ & & & \\
\hline
\end{tabular}

nachzuverfolgen. Am Universitätsklinikum Münster werden in der Standardübersicht des OP-Plans einer Klinik oder eines OP-Bereichs einzelne Operationen als Säulen dargestellt. Der Status eines jeden Patienten ist durch z.T. dokumentationsabhängige Farbmarkierungen online für alle Beteiligten ersichtlich.

Der „elektiv“ geplante Patient erscheint zunächst weiß. Sobald er von den Anästhesisten prämediziert wurde, wechselt die Farbe in hellblau, sodass auf einen Blick zu erfassen ist, welche Patienten noch nicht anästhesiologisch aufgeklärt worden sind. Der als „dringlich“ angemeldete Eingriff ist orange und die „Notfalloperation“ rot markiert. Der von der Station abgerufene Patient wird dunkelblau markiert, der Beginn der Säulenzeit gelb, das Ende der Säulenzeit hellgrün und die komplett abgeschlossene OPDokumentation dunkelgrün. Eine OPDokumentation ohne vorher erstelltes OP-Planungsformular erscheint grau. Die geplante Säulenzeit ist als schmaler Balken der tatsächlichen gegenübergestellt, sodass Planabweichungen unmittelbar erkannt werden können.

Entsprechende Übersichtsdarstellungen können einen OP-Koordinator bei den tagesaktuell erforderlichen Umstellungen eines OP-Programms unterstützen (Abb. 1).

\section{OP-Management}

Unter dem Begriff „Management“ kann nach Malik [1] das Entwickeln und Steuern produktiver sozialer Systeme ver- standen werden. Für das OP-Management umfasst dieses soziale System - in variablem und für jedes Krankenhaus individuell zu vereinbarendem Ausmaß die Mitarbeiter der an Operationen mittel- oder unmittelbar beteiligten Berufsgruppen und die von ihnen genutzten Ressourcen. Diese umfassen u.a. Räume, Geräte, Instrumente, Verbrauchsmaterialien und Medikamente. Die sich aus dieser abstrakten Definition ergebenden möglichen konkreten Aufgaben eines OP-Managers wurden von Welk umfassend herausgearbeitet [2] (Tab. 1).

Im Gegensatz dazu umfassen die Aufgaben des OP-Koordinators im Wesentlichen die Ablaufkoordination des Tagesgeschäfts. Inwieweit der OP-Koordinator dem OP-Manager zuarbeitet bzw. in die Aufgaben des OP-Managers eingebunden wird, kann den Gegebenheiten des betroffenen Krankenhauses entsprechend individuell vereinbart werden.

\section{Kommunikation}

Eine zentrale Bedeutung im OP-Management kommt der Kommunikation zu. So müssen insbesondere die vereinbarten Kennzahlen und die damit in Verbindung stehenden Leistungsdaten zeitnah nicht nur der Krankenhausleitung und den Chefärztinnen und Chefärzten, sondern allen am Prozess beteiligten Berufsgruppen verfügbar gemacht werden.

Am Universitätsklinikum Münster geschieht dies derzeit auf 3 Wegen. Zum einen gibt es den monatlichen schriftlichen gemeinsamen Bericht des Medizin- und
OP-Managements an die Krankenhausleitung und die Klinikdirektoren/innen. Zum Zweiten organisiert das OP-Management in den operativen Fachabteilungen im Abstand von 4-12 Wochen interdisziplinäre und berufsgruppenübergreifende OP-Besprechungen, deren Protokolle im Intranet einsehbar sind. Die Abstände der OP-Besprechungen variieren, je nachdem wie groß der Gesprächs- und Handlungsbedarf in einer Klinik ist. Zum Dritten stehen wesentliche Leistungsdaten und Kennzahlen tagesaktuell online im Intranet zur Verfügung.

Durch diese aktive Verbreitung und Diskussion von Zahlen, Daten und Fakten schafft das OP-Management die Voraussetzung, um mit allen Beteiligten in einem kontinuierlichen Verbesserungsprozess aus den verfügbaren Daten die individuell relevanten Kennzahlen festzulegen und daraus Zielwerte zu vereinbaren. In eine solche Kommunikation müssen alle beteiligten Disziplinen und Berufsgruppen einbezogen werden, da die unter den gegenwärtigen gesundheitsökonomischen Rahmenbedingungen erforderliche alljährliche Leistungssteigerung nur gemeinsam erbracht werden kann (Abb. 2).

\section{Typische Konflikte}

Beispiel 1: In einer operativen Fachabteilung gab es täglich Auseinandersetzungen, weil der 1 . Patient des Tages nicht wie von dem zuständigen Anästhesisten und der Anästhesiepflegekraft erwartet um 7:30 Uhr in der Einleitung war, son- 


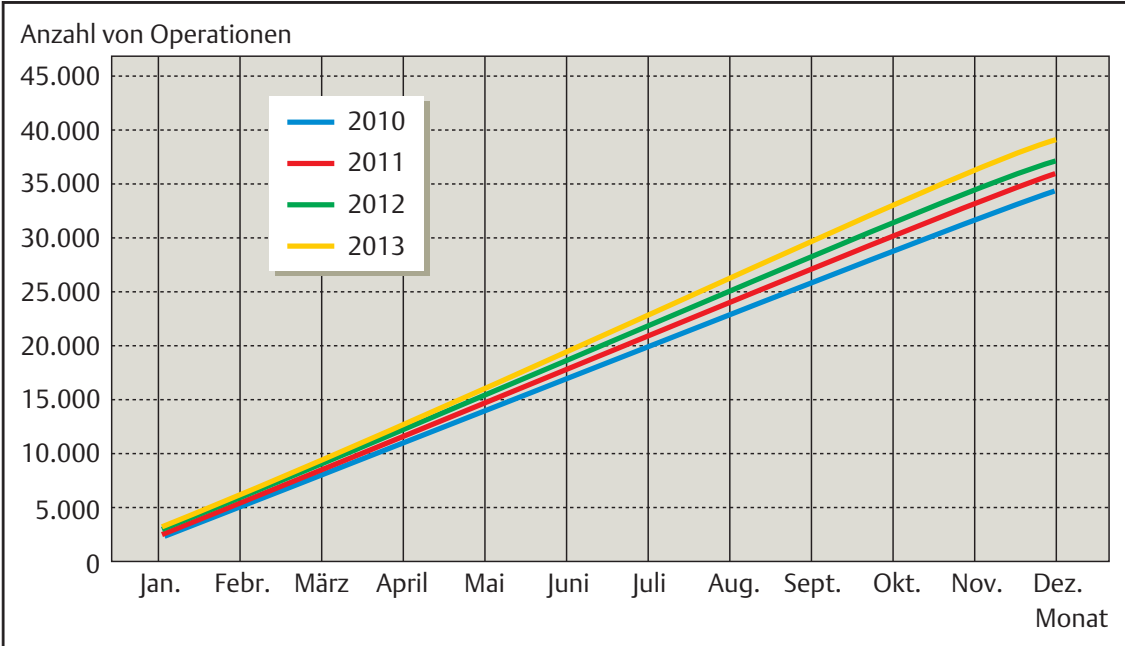

Abb. 2 Kumulative Entwicklung der Anzahl von Operationen am UKM in den Jahren 2010 bis 2013.

dern erst gegen 8:00 Uhr. Damit konnte der von den Chirurgen erwartete Zeitpunkt des 1 . Schnittes nicht eingehalten werden. In einer der ersten OP-Besprechungen dieser Fachabteilung, an der Chirurgen, OP- und Anästhesiepflegedienst, der zuständige Oberarzt der Anästhesie und Pflegepersonal aus der Ambulanz und von der Regelpflegestation anwesend waren, wurde dieser Konflikt thematisiert. Die Ambulanz- und Stationspflegekräfte stellten kurz und bündig fest, dass der 1 . Patient regelhaft für 7:15 Uhr einbestellt würde und Aufnahme, Umziehen und Prämedikation so viel Zeit in Anspruch nähmen, dass der Patient niemals bis 7:30 Uhr im OP sein könne. Es wurde vereinbart, den 1. Patienten zukünftig für $6: 45 \mathrm{Uhr}$ einzubestellen und das Problem war gelöst. So profan gestalten sich sicher nur wenige Maßnahmen zur Prozessoptimierung, aber dieses Beispiel zeigt, dass eine strukturierte Kommunikation die wesentliche Grundlage eines kontinuierlichen Verbesserungsprozesses ist.

Der Zeitpunkt des 1. Schnittes am Morgen ist ein Klassiker unter den Konflikten im OP. Wir konnten in unseren OPBesprechungen feststellen, dass die Ursachen für Verzögerungen vielfältig sind. Als Probleme erkannt und nachfolgend optimiert wurden in den verschiedenen Kliniken:

- die Stabilität des OP-Plans in dem Sinne, dass so geplant wird, dass am Morgen des OP-Tags eine Umstellung der 1. Patienten eines jeden Saales nur in Ausnahmefällen erforderlich werden kann

- der Einbestellzeitpunkt des 1. und 2. Patienten
- ein OP-Plan, der 2 Patienten von der gleichen Station zur gleichen Zeit vorsieht, obwohl die Patienten nur nacheinander in den OP transportiert und eingeschleust werden können

- die Reihenfolge der Transporte in Abhängigkeit davon, wie aufwendig die Narkoseeinleitung ist (Kombinationsanästhesie vor Allgemeinanästhesie)

- Priorisierung der Transporte in den OP gegenüber anderen Patiententransporten

- Vollständigkeit der präoperativ erforderlichen Befunde

Ein zweiter Klassiker unter den Konflikten im OP ist die Diskussion um „NahtSchnitt-Zeiten“. Ein großer Schritt zur Versachlichung dieser Auseinandersetzung ist die getrennte Betrachtung der einzelnen Komponenten dieses Zeitraums, für deren Dauer unterschiedliche Berufsgruppen und Disziplinen verantwortlich sind. So sind für die Zeit von „Naht“ bis „Ende chirurgischer Maßnahmen" noch die Chirurgen, danach bis zur „Ende Säulenzeit“ - zumindest, wenn in dieser Zeit auch die Ausleitung der Narkose erfolgt - die Anästhesisten verantwortlich. Die Dauer des sich anschließenden Zeitabschnitts, währenddessen sich kein Patient mehr im OP-Saal befindet, hängt vom Reinigungspersonal und - sofern die Instrumentiertische im Saal vor der Ankunft des nächsten Patienten gerichtet werden - vom OP-Pflegepersonal ab. Ist der OP-Saal für den Patienten vorbereitet, liegt die Zeitspanne bis zum Beginn der Säulenzeit in der Verantwortung des Anästhesisten, die anschließende Lagerung, Hautdesinfektion und das Abdecken in der des OP-Pflegepersonals und/oder der Chirurgen. Erst wenn die Dauer jeder dieser Zeitspannen bekannt ist und einzeln unter Berücksichtigung der verfügbaren (personellen) Ressourcen analysiert werden kann, ist es möglich, mit den jeweils Verantwortlichen Zielvereinbarungen über die zukünftigen Zeitspannen zu schließen. Bei undifferenzierter Betrachtung ist absehbar, dass das Thema „Naht-SchnittZeit“ eine ewige Quelle für Konflikte bleibt.

Ein dritter Klassiker unter den Konflikten im OP ist die Diskussion um die von den Chirurgen angegebenen geplanten OP-Zeiten. Sind die Abweichungen zu den tatsächlichen Zeiten regelmäßig so groß, dass die vereinbarten Saalbetriebszeiten überzogen oder Patienten sogar abgesetzt werden, sind Auseinandersetzungen vorprogrammiert.

Durch die Erfassung und regelmäßige Rückmeldung der geplanten und tatsächlichen Säulenzeiten der einzelnen Operationsarten durch das OP-Management an die Chirurgen wird die Grundlage geschaffen, die OP-Planung kontinuierlich zu optimieren.

Am UKM konnte durch diese Art der Rückmeldung die Abweichung der durchschnittlichen Säulenzeit von 12,8\% in 2010 auf 3,3\% in 2013 reduziert werden. Mittlerweile werden in mehreren Kliniken die Planungszeiten der „Standardoperationen“ in den elektronischen OP-Planungsformularen hinterlegt und regelmäßig aktualisiert.

\section{Arbeitszeitmodelle}

Von großer Bedeutung für die Mitarbeiterzufriedenheit in allen Berufsgruppen - und insbesondere in Hinblick auf die Anforderungen jüngerer Mitarbeiter der „Generation Y“ an ihren Arbeitgeber sind Arbeitszeitmodelle, die sowohl den Ansprüchen an die „Work-Life-Balance“ der Mitarbeiter als auch den betriebswirtschaftlichen Anforderungen der Krankenhausleitungen entsprechen. In einer eigenen Umfrage unter den deutschen Universitätskliniken im Frühjahr 2013, an der 18 von 34 Standorten teilnahmen, kam heraus, dass in etwa einem Drittel aller OP-Säle auch nach 15:00 Uhr regelhaft operiert wird. Im weiteren Verlauf des Nachmittags nimmt diese Anzahl kontinuierlich ab. Nach 20:00 Uhr wurde an keinem der teilnehmenden Standorte regulär operiert (Abb. 3). 


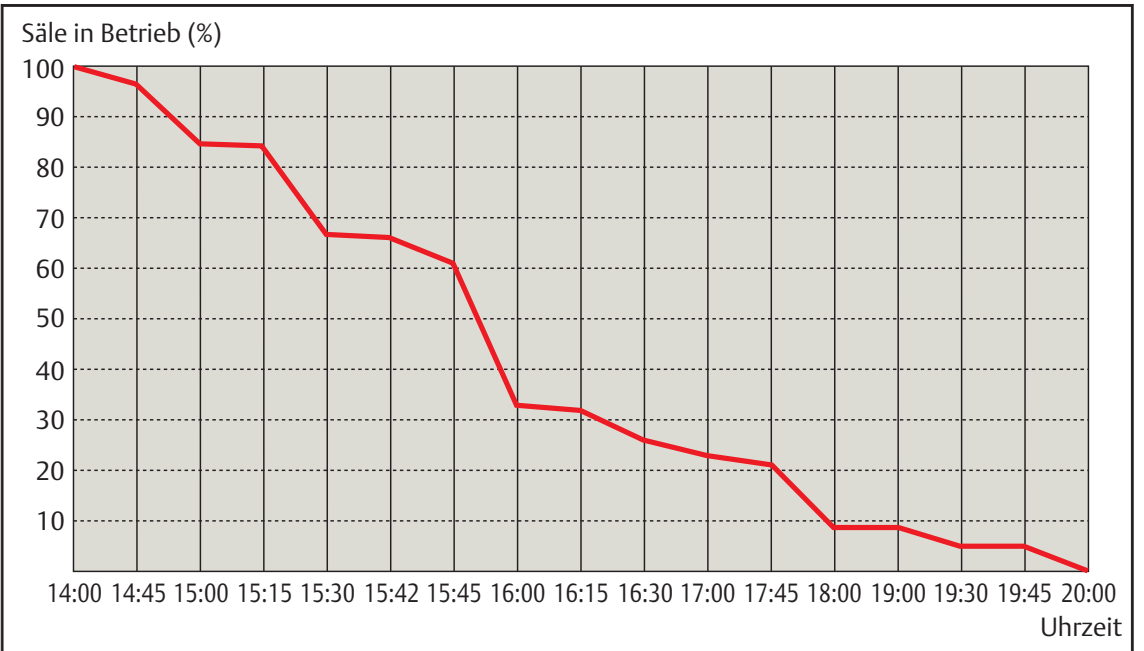

Abb. 3 Prozentualer Anteil der geplant betriebenen OP-Säle im Tagesverlauf an deutschen Universitätskliniken 2013. Bei den Uhrzeiten handelt es sich um die berichteten Endzeiten der OPKapazitäten (syn. Blockzeiten).

Die permanente Evaluation der in Anspruch genommenen OP-Kontingente und deren Auslastung gehört zu den grundlegenden Aufgaben des OP-Managements, auch im Hinblick auf die Festlegung der tatsächlich erforderlichen OP-Kontingente und die damit verbundenen Arbeitszeitmodelle.
Eine solche wiederholt durchgeführte, bedarfsadaptierte Anpassung der Arbeitszeiten gemeinsam mit der oben beschriebenen verbesserten OP-Planung hat dazu geführt, dass für den OP-Pflegedienst im Zentral-OP des UKM z.B. von April-Juni 2013 nur 0,8\% aller dokumentierten Arbeitsstunden Überstunden waren.

\section{Literatur}

1 Malik - management zentrum st. gallen in: 6 . BDA/BDC-Fortbildungsreihe „OP-Manager/ in“ - Modul 3 -; 2007

2 Welk I. Implementierung des OP-Managements. In: Welk I, Bauer M, Hrsg. OP-Management: praktisch und effizient. Heidelberg: Springer; 2006: 139-148

Dr. med. René Waurick

OP-Manager, Facharzt für Anästhesiologie, Intensivmedizin, Notfallmedizin

Universitätsklinikum Münster Stabsstelle OP-Management Domagkstraße 26 48149 Münster

waurick@uni-muenster.de 